Aus der Königl. Frauenklinik in Dresden.

\title{
Vier Fälle von Schwangerschaft im rudimentären Nebenhorn des Uterus.
}

\author{
Von \\ Dr. Krull, \\ drittem Assistenzarzte. \\ (Mit 4 Abbildungen anf Tafel VIII.)
}

Seitdem Kussmaul durch sein klassisches Werk "Von dem Mangel, der Verkümmerung und Verdoppelung der Gebärmutter" im Anschluss an 12 Fälle dieser Art das allgemeine Interesse in Anspruch nahm, lenkt jetzt wieder Kehrer durch seine im Jahre 1900 erschienene Dissertation: „Das. Nebenhorn des doppelten Uterus", dargestellt im Anschluss an 82 Fälle von Gravidität und 12 Fälle von Hämotometra die besondere Aufmerksamkeit auf diese ziemlich seltene Gravidität. Es wird daher von Interesse sein, diese 82 Fälle um drei, die in der Literatur wohl noch nicht bekannt sind, zu bereichern, während ein vierter Fall (Fall 2) durch Wehle (186. Sitzung der gynäk. Gesellschaft Dresden, 1893) zur Kenntniss kam, von Kehrer in seiner Arbeit erwähnt worden ist und an dieser Stelle eingehend beschrieben werden soll.

Sämmtliche 4 Fälle sind in der Kgl. Frauenklinik zu Dresden zur Operation gekommen.

\section{Fall I.}

Am 6. 2. 1889 wurde die 26 Jahre alte Frau des Zimmermanns K. der Klinik überwiesen. Als Kind hatte sie ausser Masern keine Krankheit gehabt, vom 17. Jahre an hatte sie regelmässig alle 4 Wochen ihre Regel, ohne Beschwerden und nicht stark. Verheirathet war sie seit dem Jahre 1887. 
Nachdem sie jm September 1888 empfangen hatte, stellten sich 3 Monate später Magen- und Leibschmerzen, Erbrechen und Durchfall ein, Beschwerden, welche sich bis zum Februar 1889 derartig steigerten, dass sie 4 Wochen zu Bett liegen und ärztliche Hülfe in Anspruch nehmen musste. Als sie dann das Bett wieder verlassen konnte, fühlte sie sich jedoch niemals schmerzfrei, auch hörten Erbrechen und Durchfall nie vollständig auf. Als Ende Juni 1889 die Entbindung zu erwarten war, stellten sich genannte Beschwerden von neuem ein, sehr starkes Erbrechen, Leibschmerzen, Durchfälle, Fieber und Harndrang, während die seit April bestehenden Kindesbewegungen aufhörten. Die Kranke lag 4 Wochen wieder zu Bett und verlor in dieser Zeit unter wehenähnlichen Schmerzen und 14 tägiger Blutung fleischähnliche Hautstücke. Der im Juni zugezogene Arzt, erklärte den Zustand für Schwangerschaft am Ende des 10. Monats. Pat, welche inzwischen sehr schwach geworden und abgemagert war, wurde Anfang September von einer zweiten 8 tägigen Blutung befallen, wobei wieder dieselben häutigen Massen abgingen, doch war sie dabei so leidlich wohl, dass sie das Bett nicht 20 hüten brauchte. Aber schon Ende September fühlte sie sich wieder schlechter, hatte häufiges Frösteln, grosse Abgeschlagenheit der Glieder und bemerkte, dass die Brüste abnahmen und reichlich Milch absonderten. Ein herzugerufener. Arzt erklärte, dass das Kind todt sei. Von October 1889 an kehrte das Wohlbefinden allmählich zurück, der Leib wurde zusehends kleiner und alle Schmerzen verschwanden. Am 20. December 1889 trat die Regel von neuem ein, ziemlich stark, 5 Tage lang und erschien zum letzten Male Ende Januar wie gewöhnlich.

Status präsens: Frau K. ist von gesundem Aussehen, Herz, Lungen und Verdaung in Ordnung. Die Brüste sondern reichlich ab. Warzenhofdrüsen deutlich vorhanden. Der grösste Leibesumfang in Nabelhöhe $83 \mathrm{~cm}$, handbreit unter demselben $98 \mathrm{~cm}$. Abstand vom Schwertfortsatz bis Nabel $21 \mathrm{~cm}$, vom Nabel bis zur Schamfuge $28 \mathrm{~cm}$. Auffallend stark ist die Pigmentirung der weissen Linie, der ganzen Bauchhaut und der Schwangerschaftsstreifen.

Das Ergebniss der in Narkose vorgenommenen inneren Untersuchung ergab:

Scheide, Scheidentheil und Gebärmutter mässig aufgelockert, letztere schlank und etwas schmal nach rechts hinten gelagert, lässt sich mit einer Kugelzange leicht hinunterziehen, wodurch man vom Mastdarm aus den rechten Eileiter und Eierstock sehr deutlich abtasten kann. An der Stelle, wo man das linke Ligament. ovar. anzunehmen hat, findet sich ein ungefähr $3 \mathrm{~cm}$ breiter Strang, welcher unmittelbar auf die Geschwulst übergeht. Auf dieser Seite lässt sich aber weder der Eierstock, noch die Tube, noch das runde Mutterband nachweisen. Die Bauchdecken scheimen zum grössten Theil mit der Geschwulst unterhalb des Nabels verwachsen zu sein. Ihre linke Hälfte wird von einem harten Kindesthei] eingenommen, in welchem knisternde Kopfknochen unverkennbar sind.

Auf Grund dieses Befundes, namentlich weil der Uterus schlank, schmal und sich sehr leicht und weit voln der Geschwulst herabziehen lässt und die rechtsseitigen Anhänge ganz klar nachweisbar sind, wird die Diagnose auf Schwangerschaft im linken mangelhaft entwickelten Nebenhorn mit abgestorbener Frucht gestellt und am 10. 2. 
1890, also 8 Monate nach dem Ende der Schwangerschaft zur Operation geschritten.

Nach Eröffnung des Leibes werden eine Menge zum Theil fester Verlötbungen zwischen Bauchwand und Geschwulst stumpf getrennt; worauf sich die ganze Geschwulst aus der Bauchhöhle wälzen lässt. Nun zeigte sich, dass die Geschwulst von dem hochschwangeren linken Nebenhorn gebildet war, denn das runde Mutterband ging aussen und unten von ihr ab, parallel mit diesem Bande lief weiter nach aussen hin die geschlängelte Tube und auf dem hinteren und unteren Abschnitt der Geschwulst lag der breitgezogene Eierstock, während am unteren Pol der Geschwulst ein vierfingerbreiter Strang zur linken Kante des rechten Hornes ging. Tube und Eierstock der rechten Seite waren völlig normal. Im rechten Eierstock befand sich ein grosses Corpus luteum.

Es wurde num ein Unterbindungsfaden um die Gefässe der Spermatica, dann ein zweiter um das runde Mutterband gelegt, ein dritter, vierter und fünfter unterband den Verbindungsstrang, worauf die ganze Geschwulst, ohne den Fruchtsack zu verletzen, abgetragen wurde. Sämmtliche Stïmpfe wurden noch einmal durch Unterbindungsfäden gesichert. Da aber die Schnürstücke ziemlich breit und umfangreich waren und der linken Uteruskante sehr dicht aufsassen, so quoll zwischen ihnen immer und immer wieder Blut hervor, namentlich blutete es aus der angerissenen Serosa unaufhaltsam weiter. Nach verschiedenen Versuchen der Blutstillung und da sich auch eine Vernähung der Serosaränder der Stümpfe unzuverlässig erwies, blieb nichts anderes übrig, als den Uterus mit den Ligaturen in die Wunde zu ziehen, unter die Stümpfe um den Gebärmutterhals herum einen Gummischlauch zu legen und den Uterus nach Porro abzutragen.

Nach fieberlosem Verlaufe wurde Pat. am 12. 3. 1890, 30 Tage p. op., entlassen.

\section{Beschreibung des Präparats. (S. Taf. VIII, Abbildung 1.)}

Die Geschwulst ist reichlich mannskopfgross, $25 \mathrm{~cm}$ lang und $20 \mathrm{~cm}$ breit von birnenähnlicher Gestalt; ihrer grösster Umfang beträgt 60 , an der breitesten Stelle $55 \mathrm{~cm}$. Oberfläche zum Theil glatt, zum Theil mit Pseudomembranen bedeckt. Der zur Gebärmutter gehende Verbindungsstrang ist $4 \mathrm{~cm}$ breit und $2 \mathrm{~cm}$ dick, ganz muskulös und ohne Hohlraum. Die Wände der Geschwulst sind an einzelnen ungleichmässigen Stellen äusserst dünn und lassen Kindestbeile sehr deutlich durchfühlen.

Nachdem die ganze Geschwulst durch Gefrieren steinhart geworden war, wurde sie der Länge nach durchsägt und so glücklich zerlegt, dass die durch die ganze Länge der Wirbelsäule getroffene Frucht in zwei ziemlich gleiche Hälften auseinanderfiel.

Bei der Betrachtung zeigt sich nun, dass die linke Hälfte etwas grösser als die rechte ist, dass der Querschnitt links oben und rechts unten ein wenig von der Mittellinie abweicht. Die umhüllende Schale, welche allenthalben muskulösen Bau zeigt, ist" an den dünnsten Stellen nur $2 \mathrm{~mm}$ stark, wenn die Placenta nicht mit berücksichtigt wird.

Der Fötus, weiblichen Geschlechtes, ist anscheinend ausgetragen und vollständig in sich zusammengekrümmt. Das Kinn (K.) ruht auf dem Schwertfortsatz, Mund und Nase (N.) auf der vorderen Bauchwand. 
Die Wirbelsäule (H. w. B. w. L. w.) ist stark hufeisenförmig gekrümmt, Atlas und Vorberg sind nur $10 \mathrm{~cm}$ von einander entfernt. Die Kopfhaut ist in Falten erholen, die Scheitelbeine (Sch.) aus ibren Verbindungen mit Stirnbein und Hinterhauptschuppen getrennt und $3 \mathrm{~cm}$ unter die Stirnbeine (St.) geschoben, während sie das Hinterhauptbein (Hb.) um fast $2 \mathrm{~cm}$ überragen. Der horizontale Umfang des Kopfes hat sonach eine Verminderung von $10 \mathrm{~cm}$ erfahren. Das Brustbein (Bb.) ist durch den Druck des Kinnes s-förmig gekrümmt mit Verbiegung in dem Winkel zwischen Hals und Mundboden und der Wirbelsäule genähert. Die ganz zusammengedrückten Lungen (Lu.) bilden einen schmalen Ueberzug des weniger beeinträchtigten Herzens (He.). Die verschmälerte Leber (Le.) läuft der unteren Brust und oberen Bauchwirbelsäule parallel. Die Därme sind stark zusammengedrückt. Die Bauchhaut in einer Falte erhoben, an deren Spitze die Nabelschnur sich einsenkt (I. Na.). An den Seiten werden Bauch- und Brusthöhle durch die eng angezogenen Beine und Arme ebenfalls eingeengt. Die Zehen berühren mit ihren Spitzen die Stirn. Der linke Arm liegt in der Furche, die der linke Oberschenkel mit dem Rücken bildet, die linke Hand liegt in Klumphandstellung dem Rücken zugewandt, das Knie ruht in der Achselhöhle. Der rechte Arm verläuft nach aussen und hinten vor dem stark nach aussen gezogen Oberschenkel, die rechte Hand in scharfer Varus-Stellung. Die einheitliche Placenta (Pl.) nimmt fast zwei Dritttheile des Fruchtsackumfanges ein, liegt hauptsächlich nach hinten und unten an der rechten Seite des Fötus mit ihrem Haupttheile in der Umgebung des kindlichen Schädels. Sie ist unregelmässig länglich gestaltet und hat mehrere rundliche Anhänge, die aber durch Placentargewebe mit der Hauptmasse in Verbindung stehen. Die Nabelschnur (Na.) verläuft zwischen den Extremitäten in mehreren Schlingen, zieht sich um die rechte Hinterbacke herum und senkt sich in der Gegend der rechten Schulter in die Eihäute ein. Da sie hier zwischen Frucht and Fruchtsack liegt, erscheint sie platt gedrückt.

\section{Mikroskopische Untersuchung.}

Von dem Fötus wurden Stücke seiner Haut und quergestreiften Musculatur, ebenso Stücke von der Placenta und der Nabelschnur in Alkohol gehärtet und nach Einbettung in Celloidin in Schnitte zerlegt, welche in Alaunkarmin bez. Hämatoxylin gefärbt und in Canadabalsam eingebettet wurden. Ein Querschnitt auf die äussere Haut zeigt sämmtliche Schichten derselben mit den darunterliegenden Muskeln zwar getrübt, aber allenthalben wohl erkennbar. Namentlich heben sich die Gänge der Schweissdrüsen, die wie Bienenwaben angeordneten quergetroffenen Bündel der Hautmuskeln, ferner das Unterhautfettgewebe deutlich hervor. Auf Längsschnitten zeigen die Muskelbündel deutlich die Querstreifung, auf Querschnitten lose Aneinanderfügungen durch grosse unregelmässige Spalten. Am auffallendsten ist das Gewebe des Nabelstranges und der Nachgeburt verändert. Das erstere zeigt von seiner bekannten 

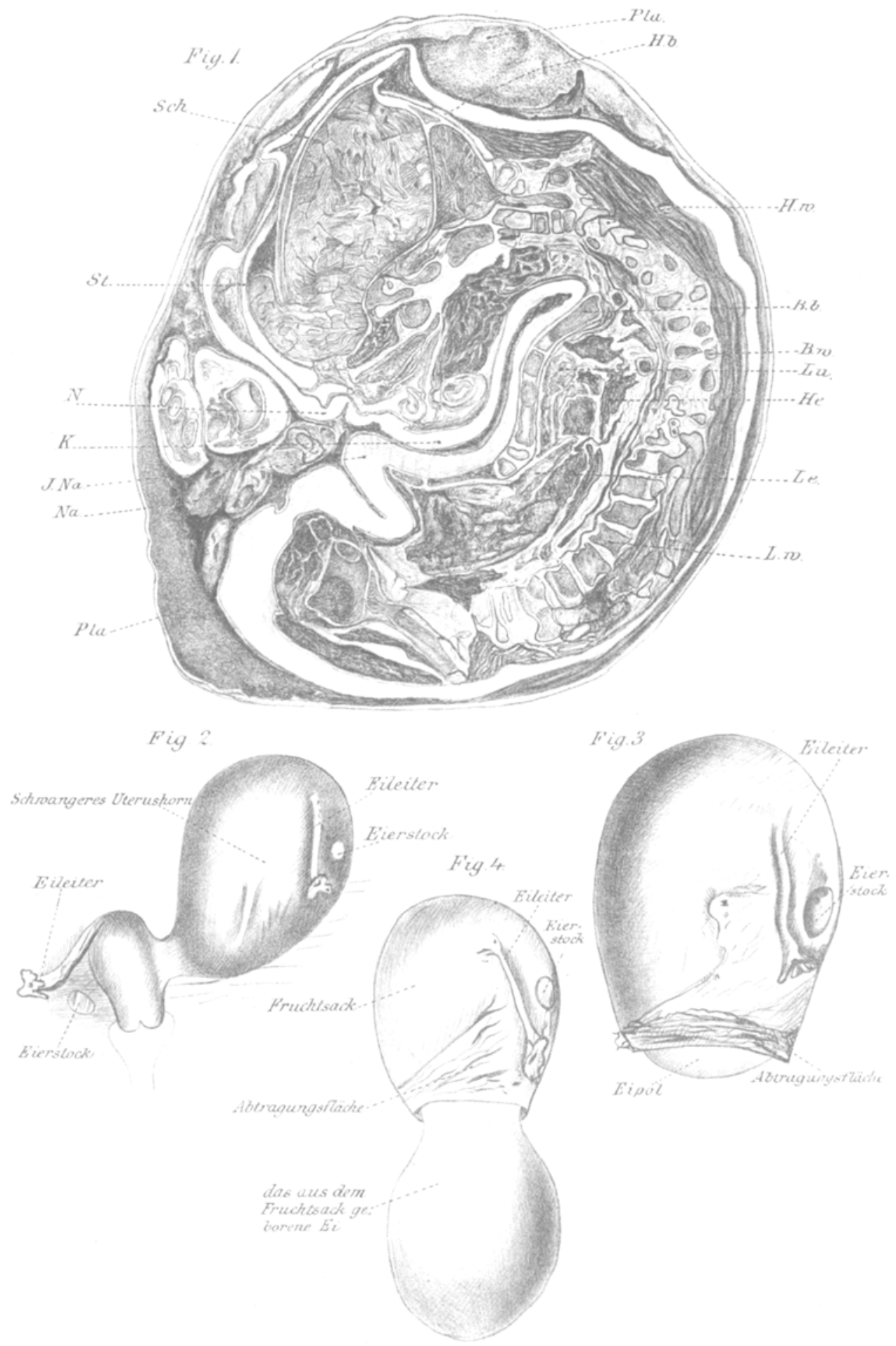
schleimigen Beschaffenheit und den sternförmigen Zellen keine Spur. Eine ausserordentlich grosse Zahl rundlicher Lücken wird ron einer trüben Grundsubstanz eingeschlossen und von den beiden Arterien und der Vene sind nur Andeutungen noch in Gestalt grosser Lücken vorhanden. In noch viel höherem Grade hat das Nachgeburtsgewebe seinen Charakter eingebüsst, indem es aus einem Durcheinander verfilzter Stränge besteht, die bräunlich getrübt sind, aber auch nicht einen eigenthümlichen Bestandtheil lebender Zotten mehr aufweisen. Auch die Lücken zwischen ihnen (intervillöse Räume) enthalten nur trübe Massen.

\section{Beurtheilung.}

Die Diagnose konnte in diesem Falle mit voller Bestimmtheit auf ausgetragene Schwangerschaft im linken rudimentären Uterushorne gestellt werden.

In hohem Grade bemerkenswerth ist der Vorgang der Befruchtung. Der Same wanderte durch das gesunde Horn und die Tube, traf ein Ei aus dem rechten Eierstock, befruchtete dies, worauf letzteres von der linken Tube aufgenommen wurde und sich im verkümmerten Horne einnistete. Es wäre auch denkbar, dass das Ei der letzten Menstruation, welches dem rechten Eierstock entstammte, schon von der linken Tube längst aufgenommen war, als der Same durch die rechte Tube erst in die Bauchhöhle gelangte. Nimmt man dann an, dass er nunmehr durch den Flimmerstrom der linken Tube zugeführt wurde und schon hier oder im verkümmerten Horn das Ei vorfand und aufgriff, so könnte auch hierfür sowohl in der Beweglichkeit als auch in der Lebensdauer der Samenfäden volle Begründung gefunden werden.

Wo sich das Ei im Nebenhorn zuerst festgesetzt hat, lässt sich nicht mehr nachweisen. Die fast Zweidrittheile des Fruchtsacks einnehmende Placenta giebt dadurch, dass sie in grössere Lappen abgetheilt ist, keinen Aufschluss. Jedenfalls war die Wand des Hornes stark genug, um dem wachsenden Ei Halt und Widerstand zu bieten, doch werden in der hierdurch bedingten Ausdehnung die Ursachen zu den Schmerzen liegen, über welche Frau R. vom dritten Monate an fast die ganze übrige Zeit zu klagen hatte. Auch die Reizung des Bauchfells, welche zu zahlreichen Verwachsungen des Tumors mit der Umgebung führte, steht mit den heftigen Schmerzen im Einklang und wird nur zu 
oft, ganz abgesehen von dem Bestehen der Schwangerschaft, das häufige Erbrechen ausgelöst haben.

Wie in anderen Fällen trat mit dem Ende der Schwangerschaft Beginn von Wehen und Abgang einer Decidua ein, auch hörten die Kindesbewegungen auf. Dem puerperalen Zustande entsprach eine monatelange Absonderung der Brüste.

In der operativen Behandlung wurde nach Abbindung des schwangeren Hornes die Erhaltung des anderen gesunden Hornes angestrebt, um der Frau fernerhin die Fortpflanzungsmöglichkeit $\mathrm{zu}$ lassen. Leider aber nöthigten die fortgesetzten Blutungen aus der linken Kante des rechten Hornes trotz aller Umstechungen und Uebernähungen der Serosa zur Unterbindung und Abtragung auch der nicht schwangeren Hälfte.

Ausnahmsweise hat in diesem Falle die Schwangerschaft ihr normales Ende ohne Ruptur erreicht. Während nun die Frucht abstarb, konnte es zur langsamen Umbildung in ein Lithopädion, zur Verjauchung, zur Maceration etc. kommen, wenn nicht die heftigen Schmerzen zur Operation geführt hätten.

\section{Fall II.}

Präparat in der 186. Sitzung der gynäkologischen Gesellschaft in Dresden von Wehle demonstrirt. (Centralblatt für Gynäkologie 1894. Jahrg. 18. S. 97.)

Am 24. 10. 1893 wurde die 35 Jahre alte Handarbeitersfrau M. aufgenommen. Als Kind hatte sie Nasern und Scharlach durchgemacht, seit Frühjahr leidet sie an heftigem Husten, dem Auswurf ist oft Blut beigemischt. 2 Geschwister sind an der Schwindsucht gestorben. Thre erste Regel hatte sie mit $20 \mathrm{Jahren}$, aufangs unregelmässig, sehr schwach, monatelang aussetzend; in den letzten 10 Jahren jedoch regelmässig. Seit 11 Jahren war sie verheirathet. Von 1883 bis 1888 hatte sie 4 mal spontan geboren, die Kinder waren ausgetragen und sind noch am Leben. Die Wochenbetten waren mit Ausnahme des letzten, wo ein Tag Fieber bestand, fieberfrei. Feh]geburten hat sie nie gebabt. Im März 1893 war die Regel zum letzten Male aufgetreten. Am 28. August entstand nach einem Fall eine Blutung, welche bis vor 9 Tagen andauerte. Anfangs gingen mehrfach Stücken ab, dann hatte sich ein übelriechender Ausfluss eingestellt. Blutung und Ausfluss liessen mach. Dafür traten aber heftige Schmerzen in Kreuz und Leib auf, dazu gesellte sich grosse Mattigkeit, Schwellung der Füsse, Appetitlosigkeit, Husten und leichter Harndrang, Beschwerden, welche auch jetzt noch bestehen.

Frau M. ist von etwas bleicher Gesichtsfarbe, und sieht elend aus. Das Herz ist ohne Krankheitsbefund, während die Untersuchung der Lungen einen Katarrh der Spitzen ergiebt. Brüste sind voll, Haut darrüber venös hyperämisch, Warzenhofdrüsen sehr zahIreich. Aus der linken Brust dentliche Sekretion. 
Der Leib ist gleichmässig aufgetrieben, links ein wenig stärker wie rechts. Die weisse Linie ist stark pigmentirt. In der linken Seite des Leibes ist eine pralle, elastische, fluktuirende, weiche, bis handbreit über die Schamfuge heraufreichende, Geschwulst zu tasten, welche sich wie eine grosse Blase mit festerem Inhalt anfühlt.

Der Scheideneingang ist injicirt, jedoch nicht deutlich venös, die Scheide ist anfgelockert, das Collum für die Fingerspitze offen, der Fundus ganz nach rechts verlagert. Die Eterushöhle ist leer, vergrössert, Körper aufgelockert und gut beweglich. Links vom Uterus setzt sich ein breiter Strang fort, der von der Gegend des inneren Muttermundes ausgeht, wie auch der Mastdarmbefund bestätigt. Die rechten Anhänge sind gut zu tasten, ebenso fühlt man links von der Geschwulst die linke Tube und das linke 0varium. Zwischen der prall elastischen, ziemlich derben Geschwulst und der Gebärmutter kommt man in eine tiefe Grube, durch die man bis zum Kreuzbein gelangen kann. Vor der Geschwulst befinden sich runde Stränge. (S. Figur 2.)

Auf Grund vorstehenden Befundes wird die Diagnose auf Schwangerschaft im linken verkümmerten Nebenhorn des Uterus gestellt und am 28. 10. die Laparotomie vorgenommen.

Operation: Nach Eröffuung des Leibes lässt sich die Geschwulst sofort herauswälzen. Ueber die Geschwulst hin läuft die linke Tube, an der äusseren Kante sitzt das linke Ovarium, wo sich ein geplatzter Follikel zeigt. Die Wand der Geschwulst ist von zahlreichen Gefässen durchzogen, von dunkelblaurother Farbe, glatt und prall gespannt. Die Geschwulst steht mit breitem festen Stiel mit dem Uterus in Verbindung. Nach Unterbindung des Eileiters und des runden Mutterbandes, nach Durchtrennung zwischen Doppelligaturen, wird ein Schlauch um den zum Uterus führenden Stiel gelegt und dieser mit kreisförmigen Messerzügen durchschnitten. Man kommt zunächst auf dicke Lagen glatter Uterusmuskulatur, schliesslich auf einen von einer prall gefüllten Blase eingenommenen Kanal, aus welchem letztere ohne Verletzung. herausgelöst wird. Der Kanal im zurückgebliebenen Stumpfe wird sondirt, er geht $2 \mathrm{~cm}$ tiefer, dann endigt er blind. Entfernung der Schleimhaut im Kanal und Verschorfung des Trichters mit dem Paquelin. Die am Stumpf zurückpräparirte Serosa wird über ihm, nach Kürzen desselben, vereinigt, der Schlanch wird entfernt und die Bauchdecken werden geschlossen.

Der Wundheilungsverlauf war ein glatter. Am 19. 11., 30 Tage p. op., konnte Pat, unter gutem Allgemeinbefinden entlassen werden.

\section{Beschreibung des Präparats.}

Ein eiförmiger Sack von $15 \mathrm{~cm}$ Länge und $8 \mathrm{~cm}$ Dicke mit glatter Oberfläche umschliesst das intakte $\mathrm{Ei}$, welches mit seiner unteren Kuppe dort aus dem Uterusborne hervorragt, wo dasselbe vom Uterus abgetragen ist. Durch die durchscheinenden Eihäute hindurch kann man ballotirende Theile des Fötus erkennen. An der äusseren linken Kante des Uterushornes befinden sich das normale Ovarium und die normale Tube der linken Seite (s. Figur 3).

Auf diese Weise stellte sich das frisch gewonnene Päparat dar, doch hat dasselbe durch Einlegen in Sublimat und dann in Alkohol seine ursprüngliche Form verloren und zwar dadurch, dass das verkümmerte Horn des Uterus, welches das Ei umschloss, sich um den 
einen Pol desselben zusammengezogen, also das $\mathbf{E i}$ aus seinem Innern nachträglich herausgeboren hat (s. Figur 4).

Dieses Präparat durch einen Schnitt in 2 Hälften zerlegt, ergiebt folgendes Bild:

Das abgetragene Uterushom ist nur noch $8 \mathrm{~cm}$ lang und $6 \frac{1}{2} \mathrm{~cm}$ dick und stark kontrahirt. Die Placenta hat ihren Sitz an der hinteren Wand, buchtet dadurch diese hervor und füllt durch ihre Dicke von $2 \mathrm{~cm}$ fast die ganze $5 \frac{1}{2} \mathrm{~cm}$ lange Höhle aus. Die Muskulatur der Wand ist an der Spitze 1 $1 / 2 \mathrm{~cm}$ dick, während sie nach der Abtrennangsfläche zu an Stärke zunimmt und hier die Dicke von $21 / 2 \mathrm{~cm}$ erreicht. Die Muskulatur selbst bietet nichts Besonderes dar, man sieht in ihr die Querschnitte zahlreicher Gefässe. Die $12 \mathrm{~cm}$ lange und ca. $11 / 2 \mathrm{~cm}$ dicke Placenta ist in dem Zustand der Lösung begriffen, sie haftet zur Hälfte noch der hinteren Uterushornfläche an, zur Hälfte ist sie geboren, während der Fötus schon das Innere des Fruchtsackes verlassen hat, mit dem Kopfe vor der Austrittsöffnung. Der Fötus ist $11^{1 / 2} \mathrm{~cm}$ lang und entspricht dem 4. Monat der Schwangerschaft. Die Nabelschnur senkt sich ziemlich central ein.

\section{Beurtheilung.}

Dass es sich in diesem Falle um eine Schwangersehaft im linken verkümmerten Uterushorne handelte, konnte mit Sicherheit angenommen werden. Während das Ausbleiben der sonst regelmässig auftretenden Regel, die Veränderung an den Brüsten, die Annahme einer Schwangerschaft wahrseheinlich machte, musste auch der innere Befund, nämlich die Auflockerung der Scheide, des Collums und des Gebärmutterkörpers diesen Verdacht bestätigen. Da jedoch die Uterushöhle leer war und sich links eine Geschwulst befand, musste zunächst an eine extrauterine Schwangerschaft gedacht werden. Allein der breite Verbindungsstrang zwischen Gebärmutter und Geschwulst ging von der Gegend des inneren Muttermundes aus, es waren die Anhänge rechts vom Uterus deutlich zu tasten, während der untersuchende Finger links nur eine tiefe leere Grube zwischen Geschwulst und Gebärmutter fühlte, in deren Tiefe man bis auf das Kreuzbein gelangen konnte. Alle diese Beobachtungen, zumal noch links oben und vorn von der Geschwulst Gebilde zu fühlen waren, welche den linken Anhängen entsprachen, mussten zur obigen Diagnose führen, welche auch durch die Operation in vollem Umfange bestätigt wurde.

Begnügt man sich mit dem Ergebniss der Sonde, welche den im Stiel befindlichen Canal blind endigen lässt, so stellt sich in diesem Falle der Gang der Befruchtung, da das Corpus luteum sich in dem linken Eierstock befindet, ein wenig anders und einfacher dar. Der Same wäre dann durch das gut entwickelte Horn 
und die zu ihr gehörende Tube in die Bauchhöhle gedrungen, traf entweder hier ein Ei des anderen Eierstockes und befruchtete dieses oder dieser Vorgang hätte sich erst in der Tube bezw. in der Höhle des verkümmerten Hornes abgespielt.

Die dünnste Stelle der Wandmuskulatur befindet sich an der Spitze, ein Befund, der sehon bei anderen Fällen häufig beobachtet worden ist und als häufigste Stelle der Zerreissung angegeben wird.

Hervorzuheben wäre noch der Verlauf der Tube auf dem schwangeren Uterushorn. Da die Placenta ihren Sitz an der hinteren Wand hat und so auch zu einer grösseren Ausbuchtung derselben geführt hat, so sind die Anhänge mehr nach vorn gedrängt worden und nehmen eine schräge Richtung von oben nach unten aussen ein, sodass, wenn ein Gegenstück auf der anderen Seite des schwangeren Hornes angenommen würde, die Anhänge divergent nach unten verliefen, eine Beobachtung, durch welche schon durch äussere Untersuchung an der Lebenden im schwangeren Uterus der Sitz der Placenta bestimmt werden kaun.

Die Blutung, welche bei Pat. nach dem unglücklichen Fall entstanden ist, hängt wohl mit dem gleichzeitigen Eintritt von Wehen zusammen. Vielleicht sind die Stücke, welche mit der Blutung abgegangen sind, auf die aus dem Uterus ausgestossene Decidua zu beziehen.

Die Art der Operation erstrebte natürlich die möglichste Erhaltung der Organe, welche auch durch Abtragen des verkümmerten Hornes und nachträglichen Verschluss des Stumpfes erzielt wurde.

Dass die Fortpflanzungsfähigkeit in keiner Weise beeinträchtigt wurde, zeigte das nächste Jahr. Am 22. 10. 1894 wurde Frau M. nach 6 stündiger Geburtsdauer von einem gesunden Knaben in hiesiger Klinik entbunden. Das Kind wurde in Schädellage II a geboren, wog $2810 \mathrm{~g}$ und war $48 \mathrm{~cm}$ lang.

\section{Fall III.}

Am 30. Mai 1899 fand die 26 Jahre alte Töpfersfrau Clara Sch. Aufnahme in hiesiger Klinik. Als Kind hatte Frau Sch. englische Krankheit und Masern gehabt, sonst war sie stets gesund gewesen. Die erste Regel trat im 15. Lebensjahre auf, seit dieser Zeit alle 4 Wochen, 5-8 Tage lang und ziemlich stark. Frau Sch. hat zweimal ausgetragene Kinder geboren. Die letzte Regel hatte sie vom 15. bis 18. August 1898, stark, die ersten Kindesbewegungen wurden Ende Januar 1899 beobachtet. Im Verlaufe dieser Schwangerschaft batte Frau Sch. öfters Schmerzen im Unterleib. Im Januar war nachts plötzlich ein so ausserordentlich heftiger Schmerz aufgetreten, verbunden mit 
dem Gefühle heftiger Bewegungen im Unterleib, dass Patientin am nächsten Morgen einen Arzt zu Rathe zog, welcher jedoch ausser dem Fehlen kindlicher Herztöne, nichts Besonderes feststellen konnte. Da die Leib- und Kreuzschmerzen in der Folgezeit nicht aufhörten, wurde ihr der Rath gegeben, die Klinik aufzusucben.

Frau Sch. ist bei der Aufnahme gut entwickelt und von kräftigem Körperbau. Herz- und Lungenbefund ergiebt keine krankhaften Veränderungen.

Die Warzenhöfe sind pigmentirt, Warzenhofdrüsen sind vorhanden und die Brüste secerniren.

Der Leib hat die Form eines Spitzbauches, der Umfang beträgt $113 \mathrm{~cm}$, der Abstand vom Schwertfortsatz bis Nabel $28 \mathrm{~cm}$, vom Nabel bis zur Symphyse $21 \mathrm{~cm}$. Der Leib ist druckempfindlich, die Haut der Unterbauchgegend ist geröthet und etwas ödematös. Fundus ut. steht handbreit über dem Nabel, Kindestheile sind schon wegen der Straffheit der Bauchdecken nicht fühlbar, ebensowenig Tuben und Ligamente, nur rechts vom Nabel ist ein Knacken zu fühlen. An den Uterus schliesst sich links, durch eine Einsenkung von ihm getrennt, eine ca. faustgrosse, pralle Resistenz an. Der Beckeneingang ist frei, kindliche Herztöne sind nicht hörbar. Auffallend ist die nach unten zunebmende Breite des Uterus. Seine Ausdehnung entspricht nicht der Grösse einer Gebärmutter bei ansgetragenem Kinde. Die innere Untersuchung ergiebt eine mässig weite, bläulich verfärbte Scheide mit weichem, aufgelockertem Collum. Das ganze kleine Becken ist leer. Der äussere Muttermund ist für die Fingerspitze durchgängig, der innere ist geschlossen. Ein vorangebender Kindestheil konnte nicht gefühlt werden.

Es wurde un zunächst angenommen, da der untersuchende Finger das Collum in das untere Uterussegment übergehend fühlte und die von aussen neben dem Uterus liegende faustgrosse Resistenz innerlich mit Sicherheit nicht festgestellt werden konnte, da seit Monaten keine Kindesbewegungen mehr beobachtet waren und man von aussen deutlich das Gefühl schlotternder Knochen hatte, dass es sich um ein im VII. Monat abgestorbenes, in sich zusammengeschrumpftes Kind in Steisssehiefiage handle und dementsprechend die Diagnose auf in trauterine Schwangerschaft gestellt.

Zur Einleitung der Geburt wurden nun Jaminaria eingelegt, doch blieben trotzdem die Wehen aus.

Die Untersuchung, die darauf in Narkose vorgenommen wurde, hatte ein ganz anderes Ergebniss. Recht's rom Nabel ist ein kindlicher Kopf mit schlotternden Knochen zu fühlen. Der Finger, welcher jetzt durch den inneren Muttermund kommt, findet eine leere Uterushöhle ror. Der Uterus ist nach links verschoben, etwas über der Symphyse, jhm von hinten aufliegend, eine bis handbreit über den Nabel reichende Geschwulst. Zwischen dieser und der Gebärmutter spannt sich ein fester, derber Strang.

Nach diesem Befunde wurde die Möglichkeit einer rechtsseitigen Nebenhornschwangerschaft angenommen und am 10. Juni die Laparotomie ausgeführt.

Nach Eröffnung des Leibes werden einige Verlöthungen des Netzes mit der Geschwulst unterbunden und durchtrennt. Es erscheint nun der Fruchtsack, dessen morsche Wand bei Berührung sofort aufbricht. Die Zerreissungsstelle wird vergrössert und die mit dem Kopf sichtbare Frucht an diesem extrahirt, nachdem die Umgebung mittelst Tücher 
vor dem Fruchtwasser geschützt wird. Die Nabelschnur wird durch. tremnt. Nach Durchtrennung einzelner Verwachsungen wird der Frucht_ sack herausgewälzt, es ist das dem Uterus nach rechts aufsitzende gra vide Nebenhorn. Nach Abbindung des Stieles, des runden und breiten Mutterbandes in $3 \mathrm{Abtheilungen,} \mathrm{werden} \mathrm{die} \mathrm{einzelnen} \mathrm{Ligaturabschnitte}$ mit dem Paquelin durchtrennt, jeder Abschnitt wird mit einer Sicherheitsabschnürung versehen und der Stumpf nach Umstechung einiger blutender Spermaticaäste versenkt. Der Uterus war vergrössert und aufgelockert und lag bei der Herauswälzung des Fruchtsackes links von demselben; die Anhänge auf der linken Seite waren normal. Nach Abtragung einiger durch Fruchtwasser grünlich verfärbter Verwachsungen zwischen Bauchwand und Fruchtsack und nach Entfernung eines Theils der überschüssigen Bauchbaut mit dem Nabel wird der Bauch geschlossen.

Der Blutverlust war relativ mässig, doch erhielt Patientin trotzdem 200 gr Kochsalzlösung infundirt.

Der Verlauf der Heilung verlief ohne Störung. Am 2. 6. konnte Frau Sch. mit ivollkommen frei beweglichem Uterus bei bestem Woblsein aus der Klinik entlassen werden.

\section{Beschreibung des Präparats.}

Der eröffnete Fruchtsack ist bis auf die Grösse eines Strausseneies zusammengezogen. Die obere vordere Fläche ist mit derben Adhäsionen besetzt, die den Verwachsungen mit der Bauchwand entsprechen. Hier befindet sich auch die runde erweiterte Rupturstelle, deren Durchmesser am gehärteten Präparate etwa $11 \mathrm{~cm}$ beträgt. Entgegengesetzt von der Rupturstelle befindet sich die doppeldaumendicke Abtrennungsstelle vom Uterus. Von dieser Abtremnungsstelle $10 \mathrm{~cm}$ entfernt auf der vorderen Seite des Tumors entspringt die $12 \mathrm{~cm}$ lange, gerade laufende bis bleistiftdicke Tube mit offenem Lumen und erhaltenem Fimbrienende. Auch die Fimbria ovarica ist erhalten. Das Ovarium ist glatt, $6 \mathrm{~cm} \mathrm{lang,}$ $2^{1 / 2} \mathrm{~cm}$ breit, $1^{1 / 2} \mathrm{~cm}$ dick. Der Durchschnitt des Ovariums zeigt kleine Cysten und ein Corpus lnteum verum. Von'der Abgangsstelle der Tube aus zur Abtrennungsfläche vom Uterus zu verlaufend, findet sich ein $5 \mathrm{~cm}$ langer Rest des Ligament. rot.

Unter dem serösen Ueberzug des Fruchtsackes findet sich eine Muskelschicht von $1 / 2-1 \frac{1}{2} \mathrm{~cm}$ Dicke, die von zahlreichen Gefässen durchsetzt ist. Mit dieser Schicht fest verwachsen sitzt im ganzen Bereich der Innenwand des Fruchtsackes die gut entwickelte Placenta. Die Fruchthöhle am gehärteten Präparat ist $12 \mathrm{~cm}$ lang, $7 \mathrm{~cm}$ breit und $6 \mathrm{~cm}$ tief. Die ganze Geschwulst ist stark contrahirt, so dass sich die Placenta in dicken Wülsten, tiefe Buchten bildend, gegen die Fruchthöhle hervorhebt. Die fötale Fläche der Placenta ist von den Eihäuten überkleidet. Die Nabelschnur inserirt in der Mitte der vorderen Wand, sie ist $40 \mathrm{~cm}$ lang und zeigte im frischen Zustande deutliche Zeichen der Fäulniss.

Das Kind, männlichen Geschlechts, war stark macerirt. Seine Länge beträgt $50 \mathrm{~cm}$, es ist $2270 \mathrm{gr}$ schwer.

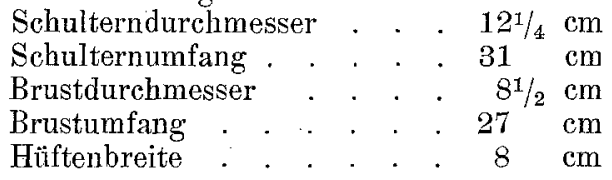




\begin{tabular}{|c|c|c|c|c|}
\hline Gerader & Kopfdurchme & esser. & $11^{3 / 4}$ & \\
\hline Senkrech & iter & & $9^{3} / 4$ & $\mathrm{cH}$ \\
\hline Grosser & querer " & & $9^{1 / 2}$ & \\
\hline Kleiner & querer $"$ & & 8 & $\mathrm{~cm}$ \\
\hline Schräger & $\eta$ & & $13^{1 / 4}$ & 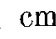 \\
\hline Grösster & Umfang des & Kopfes & 36 & $\mathrm{~cm}$ \\
\hline Mittlerer & $\eta$ & $"$ & 34 & \\
\hline Kleinster & $n$ & $"$ & 32 & \\
\hline
\end{tabular}

Besehreibung des mikroskopischen Präparats.

Querschnitt durch den Stiel nach dem Uterus. Es wurden Stücke in Formalin-Alkohol gehärtet, in Paraffin eingebettet und die Schnitte mit Hümatoxylin-Eosin gefärbt. Man erhält folgendes Bild: Nach aussen finden sich nur an einzelnen Stellen erhaltene Serosareste. Im Uebrigen zeigt der ganze Schnitt aufgelockerte, längs und quer verlaufende Muskelbündel mit reichlichem Zwischengewebe, das stellenweise kleinzellig infiltrirt ist.

Zwischen den Muskelbündeln sieht man schon mit blossem Auge zwei feine Oeffnungen, in deren Umgebung die Musculatur eine deutlich concentrische Anordnung zeigt. Mikroskopisch findet man diese Lumina mit einem einschichtigen Cylinderepithel ausgekleidet. Eine typische Submucosa fehlt. Die Schleimhaut des Canals, dessen Lumen in dem Querschnitt zweimal getroffen erscheint, springt in einzelnen Falten vor, an einzelnen Stellen gewinnt man den Eindruck, als ob es sich un den letzten Rest mit Cylinderepithel ausgekleideter Drüsen handelt. Der ganze Befund spricht für den noch vorhandenen, aber stenosirten Verbindungsgang zwischen rudimentärem Uterushorn und der normalen Uterushöhle. Die Muskulatur in der Umgebung dieses Canals zeigt eine deutliche concentrische Schichtung.

\section{Beurtheilung.}

Wenn in diesem Falle im Anfange die Diagnose auf Schwangerschaft im rudimentären Uterushorne nicht erkannt ist, so lag es an der Ungunst der Verhältnisse. Die Klagen der Patientin konnten allein auf den Zustand der Gravidität zurückzuführen sein, der Befund der äusseren und inneren Untersuchung konnte zunächst nicht auf das Vorhandensein einer ektopischen Schwangerschaft hinweisen, alle Unstände sprachen dafür, dass es sich nur um ein in der Schwangerschaft abgestorbenes, im normalen Uterus befindliches Kind handeln konnte, weshalb die Einleitung der Geburt angezeigt erschien. Die leere Uterushöhle nach Erweiterung des Cer- 
vicalcanals mit Laminariastiften zerstörte natürlich die ganze obige Diagnose und der in Narkose festgestellte derbe Strang zwischen Uterus und Geschwulst wies mit grösster Wahrscheinlichkeit auf das Bestehen einer Nebenhornschwangerschaft hin.

Auch hier wie im Falle I handelte es sich um eine Schwangerschaft im verkümmerten Nebenhorne am Ende der Zeit, die Vornahme der Operation stimmt ungefähr mit dem Zeitpunkt der fälligen Geburt überein. Dass schon im Januar keine Herztöne des Kindes mehr beobachtet wurden, beruht wohl auf einem Irrthum, das Kind kann zu dieser Zeit noch nicht ausgetragen gewesen sein. Der Tod des Kindes wird in die Zeit der letzten Untersuchung des Arztes, welcher Patientin in die Klinik schickte, fallen.

Die Schmerzen, über welche Patientin im Verlaufe der Schwangerschaft zu klagen hatte, lassen sich durch die Ausdehnung des Fruchtsackes und durch die zahlreichen Verwachsungen desselben mit dem Netz und der Bauchwand und durch die damit bedingten Zerrungen erklären.

Dass die Spitze des schwangeren Uterushornes vor allen Dingen den Gefahren der Zerreissung ausgesetzt ist, zeigte sich auch in diesem Falle, die beginnende Usur konnte deutlich am obersten Pol beobachtet werden.

Die Befruchtung kann vielleicht in diesem Falle auf dem nächsten Wege erfolgt sein. Weshalb sollte man auch nicht annehmen, dass hier der Same direkt dureh den vorhandenen Verbindungsgang des verkümmerten Hornes gedrungen ist und ein $\mathrm{Ei}$, entstammend dem Eierstock derselben Seite, befruchtet bat. Denn das Lumen dieses Canals, das man mit blossem Auge schon erkennen kann, ist sicher weit genug gewesen, um eindringende Samenfäden passiren zu lassen.

Die Operation war auch in diesem Falle so conservativ wie. möglich, wenn auch die Blutstillung aus dem ziemlich breiten Stumpfe sehr erschwert war.

Wie im vorigen Falle, so wurde auch Frau Sch. wieder schwanger und in hiesiger Klinik am 4. August 1900 von einem gesunden Mädchen entbunden. Das Kind wurde spontan in Steisslage geboren, wog $3430 \mathrm{~g}$ und war $50 \mathrm{~cm}$ lang. Die Geburtsdauer währte 7 Stunden. 


\section{Fall IV.}

Frau Clara P., Feuerwehrmannsehefrau, 22 Jahre alt, fand am 12. 1. 1900 , nachmittags 4 Uhr 30 Min., Aufnahme in der Klinik. Ausser Diphtherie und Bleichsucht will Pat, keine weitere Krankheit gehabt haben. Im 16. Jahre trat zum ersten Male die Regel auf, seitdem alle 4 Wochen 3 bis 5 bis 8 Tage lang, mittelstark. lhre letzte Regel hatte sie Mitte September. Verheirathet war sie seit 1889, jetzt war sie zum ersten Male schwanger.

Frau P. hatte am 11. 1. 1900 einen Kinderwagen mit Wäsche gefahren und hiermit einen Weg von $2^{1 / 2}$ Stunden zurückgelegt. Sie kam mittags um 12 Uhr an ihrem Bestimmungsort bei bestem Wohlsein an. Um 2 Uhr nachmittags aber überfiel sie plötzlich eine solche Mattigkeit, Schwindel und Ohnmachtsgefühl, dass sie umsank. Der herbeigerufene Arzt stellte eine innere Blutung, wahrscheinlich ausgehend von einer geplatzten Eileiterschwangerschaft, fest und rieth der Frau, sofort die hiesige Klinik aufzusuchen. Dies geschah aber erst am nächsten Tage. In der Zwischenzeit fühlte die Pat. sich sehr matt und hinfällig. Die Ueberführung geschah in einem Wagen, in welchem die Pat. aufrecht sass.

Frau P. ist übermittelgross, von schwächlichem Körperbau und in dürftigem Ernährungszustande, von ausserordentlich anämischem Aussehen. Die Herzgrenzen sind normal, der erste Ton über der Mitralis ist von einem systolischen Geräusch begleitet. Die Lungen sind ohne pathologischen Befund. Die Brüste sind gut entwickelt und zeigen geringe Sekretion.

Der Puls ist sehr klein, die Respiration ist mühsam, die Mattigkeit bedeutend, der Leib ziemlich druckempfindlich.

Nachdem die Diagnose auf geplatzte Tubargravidät gestellt war, wurde sofort die Laparotomie in Aethernarkose angeschlossen, gleichzeitig erhält Pat. subcutan eine Kochsalzinfusion von $500 \mathrm{~g}$.

Bei Eröffnung der Bauchböhle sieht man in ihr schwimmend den Fötus, entsprechend dem 4.-5. Monat, in den unverletzen Eihüllen, welcher in toto entfernt wird. Um den Stiel des geplatzten Fruchtsackes, welcher dem rechten rudimentären Uterushorn entspricht, werden Ligaturen gelegt, das Ligament. lat. wird abgebunden, doch so, dass bei Entfernung des gestielten Fruchtsackes Tube und Ovarium derselben Seite zurückbleibt. Nach Verschorfung des Stumpfes, nach Reinigung der Bauchhöhle von Blutgerinnseln wird der Leib geschlossen.

Frau P. erholte sich bald nach der Operation.

Am 30. 1. konnte Pat. aufstehen und am 6. 2. wurde sie bei bestem Wohlsein entlassen.

\section{Beschreibung des Präparats.}

Die Stielabbindungsfläche des rudimentären Nebenhorns ist $4: 4 \mathrm{~cm}$, ein wenig uneben, von weisslicher Farbe und mit zahlreichen Gefässöffnungen versehen. Das abgetrennte Nebenhorn besteht zunächst aus einem hühnereigrossen Stück mit deutlicher derber Muskulatur und deutlichem Serosaüberzug und geht dann mit einer unregelmässigen gezackten circulären Ruptur in einen faustgrossen, unregelmässig gestalteten Tumor über, welcher aus Placentargewebe und einem Theil des Fruchtsacks besteht. Dieser ist nur noch bis auf ein Stück von $10 \mathrm{zu}$ 
$11 \mathrm{~cm}$ erhalten, seine Innenfläche hat einen glatten spiegelnden Ueberzug. Der Ansatzpunkt der Nabelschnur ist nicht zu finden. Der Eihautsack, welcher noch vollkommen erhalten und mit hellem Fruchtwassser gefüllt ist, in welchem der Fötus schwimmt, ist vollständig getrennt von oben beschriebener Geschwulst. Der Eihautsack ist 13/2 zu $11 \mathrm{~cm}$ gross, glatt, nur mit einigen zottigen Auflagerungen versehen. Die Nabelschnur geht durch die Eihäute und ist bei ihrem Austritt abgerissen. Der Fötus ist $22 \mathrm{~cm}$ lang, und männlichen Geschlechts. In den Eihäuten ist ein linsengrosses gelblich aussehendes deutliches Nabelbläschen.

\section{Mikroskopische Untersuchung.}

Schnitte durch die Stielabbindungsfläche des verkümmerten Hornes und durch die Placenta, gehärtet in Formalin-Alkohol, eingebettet in Paraffin und gefärbt mit Hämatoxylin-Eosin.

Die muskulären Elemente haben eine starke Vermehrung und Vergrösserung erfahren. Die Muskelbündel, an denen eine regelrechte Anordnung oder lamelläre Schichtung nicht zu erkennen ist, verlaufen längs und quer, durch lockeres $Z$ wischengewebe mit einander vereinigt. Die Gefässe, die in grosser Anzahl vorhanden sind, zeichnen sich durch ihre Wandstärke aus. Entsprechend der Hypertrophie und Hyperplasie der Musculatur hat auch der seröse Ueberzug, welcher noch häufig vorhanden ist, eine Vermehrung erfahren. Am Rande des Schnittes ragt eine ca. gänsekieldicke Fläche hervor, in ihr erkennt man mit dem blossen Auge 2 Oeffnungen, die eine, mehr lateral liegend, entspricht einem grossen Gefässe, die andere ist mit einschichtigem Cylinderepithel ausgekleidet, an dem Flimmerhaare nicht mehr zu erkennen sind. Die Schleimhaut springt in wenigen Falten gegen das Lumen vor, um dasselbe eine Rings- und Längsmuskelschicht, ein Bild, wie es uns die Tube am uterinen Ende bietet.

Das mikroskopische Bild der Placenta bietet im Grossen und. Ganzen das eines intrauterinen Aborts.

Ein Verbindungsgang zwischen Horn und wirklicher Uterushöhle oder Reste eines Ganges sind nicht aufzufinden.

\section{Beurtheilung.}

Dass die Diagnose in diesem Falle auf Schwangerschaft im: rudimentären Nebenhorne nicht gestellt werden konnte, liegt auf der Hand. Die Anzeichen der inneren Blutung, die wahrscheinlichen Zeichen der Schwangerschaft liessen nur auf eine geplatzte 
Tubargravidität schliessen, so dass in der kürzesten Zeit zur Laparotomie geschritten werden musste, um die Quelle der Blutung zu stillen. Interessant ist, dass die Ruptur schon ca. 25 Stunden vor der Operation eingetreten war und dass Frau P. den mehrstündigen Wagentransport ohne Nachtheil überstand. Der Umstand, dass keine grösseren Gefässe verletzt worden sind und dass sich bald in der Ruhelage - in der Zeit zwisehen Ruptur und Transport - eine geeignete Thrombenbildung anschloss, haben. wohl eine allzu grosse Blutung verhindert.

Wie die Befruchtung erfolgt sein kann, ist in diesem Falle zu problematisch, zumal ein wichtiger Anhaltspunkt, in welchem Ovarium sich das Corpus lut. ver. befindet, bei der Operation übersehen ist.

Die Ursache der Ruptur wird in der Anstrengung zu suchen sein, welcher sich Pat. kurz vorher unterworfen hat, indem sie 2 Stunden lang einen Wagen mit Wäsche schob.

Die Ruptur ist circulär aufgetreten und zwar so, dass auf dem Stumpf wie ein Blumenstrauss die Placenta aufsass und dass der Riss dicht oberhalb des Abganges der Tube verlief. Während die Tube sonst entsprechend ihrer anatomischen Lage der Ausdehnung des schwangeren Uterus folgt und gewöhnlich von der höchsten Spitze der Seitenkante entspringt, geht die Tube des verkümmerten Hornes dicht oberhalb des Stiels ab, die Ausdehnung des schwangeren Hornes hat diese Wand ziemlich unberücksichtigt gelassen und hat sich auf die gegenüberliegenden Wände beschränkt. Es konnte deshalb ein möglichst conservatives Vorgehen im Gange der Operation erreicht werden, indem ein Doppelfaden unterhalb des $\mathrm{Ab}$ gangs der Tube, der eine um die Tube, der andere um den Stiel gelegt wurde, sodass die durchtrennte Tube mit dem Ovarium zurückblieb und nur das schwangere Horn mit der Tube am uterinen Ende - wie es das mikroskopische Bild zeigt - exstirpirt wurde.

Das Wohlbefinden der Pat. nach der Entlassung hielt an, was bei späterer Wiedervorstellung bestätigt werden konnte.

Diese 4 Fälle bieten insofern grosses Interesse dar, als bei zweien (Fall 1 und 2) die Diagnose auf Gravidität im verkümmerten Uterushorn mit voller Sicherheit und bei Fall 3 mit grösster Wahrscheinlichkeit gestellt wurde. Fall 4 liess in seiner Schwere und in der Kürze der Zeit nur die Diagnose auf geplatzte Tubargravidität zu. 
Bevor nun eine kurze Zusammenfassung dieser Fälle folgt, möchte zunächst klargestellt werden, dass es sich in unseren Fällen auch wirklich um eine Schwangerschaft im rudimentären Nebenhorn des Uterus gehandelt hat. Was ist ein rudimentäres Nebenhorn? Während man darunter ein schlecht entwickeltes und ernährtes, entweder ein hohles oder ein solides Horn eines Uterus bicornis unicollis versteht, dessen meist zu einem Stiel ausgezogener solider oder canalisirter Verbindungsstrang mit dem besser entwickelten Horne in der Gegend des inneren Muttermundes sich verbindet, so hält Kehrer diese Definition nieht für glüeklich gewählt. Mit Rücksicht auf gewisse pathologisch-anatomisehe Verhältnisse müsse man dabei 2 Gruppen unterscheiden:

I. Uterus bicornis und septus bilocularis mit relativ guter Entwicklung der Muskulatur und Gefässe des atretischen oder stenosirten graviden Nebenhorns.

II. Uterus bicornis und septus bilocularis mit eigentlichem rudimentären Nebenhorn.

Während zu der ersten Gruppe diejenigen Fälle gerechnet werden, wo erst nach dem 7. Monat eine Ruptur auftritt, wo eine Schwangerschaft das normale Ende erreicht oder wo die Frucht über den Endtermin getragen wird, werden zum eigentlichen rudimentären Horn die vor dem 7. Monat zur Ruptur kommenden Schwangerschaften gezählt. Der Begriff „rudimentär" fällt demnach nach Kehrer für Fall 1 und 3 , wo es sich um ausgetragene Früchte handelt, fort, doch giebt auch Kehrer zu, dass Ausnahmen von der Regel vorkämen, seien doch sogar Fälle zu verzeichnen, wo eine dünnwandige Tube ohne Ruptur ein ausgetragenes Kind beherbergt habe.

Bei der Aufstellung dieser Fälle jst der von Kehrer aufgestellte Unterschied nicht gemarht worden. Das Bild ist auch einheitlicher und übersichtlicher, wenn man sich der bisherigen Nomenclatur, nach der alle 4 Fälle in das Gebiet des rudimentären Hornes zu zählen sind, anschliesst. Entspricht doch der Befund bei allen 4 Fällen allen dafür aufgestellten, schon vorher erwähnten Bedingungen. Bei. allen Fällen ist ein längerer oder kürzerer Verbindungsstrang des verkümmerten mit dem besser entwickelten Horn zu finden gewesen, der Strang war entweder solid oder der Canal war stenosirt.

Bei solchem Befunde ist in hohem Maasse bemerkenswerth der 
Vorgang der Befruchtung.

Bei Fall 1, 2 und 4 war der Verbindungsstrang solid. Bei Fall 1 war das Corpus lut. auf der Seite des gut entwickelten, bei Fall 2 auf der Seite des verkümmerten Hornes. Im ersten Falle haben wir es daher mit einer äusseren Ueberwanderung des Eies und des Samens, bez. des befruchteten Eies, im zweiten Falle mit einer äusseren Ueberwanderung des Samens zu thun. Bei Fall 4 fehlt ein wichtiger Anhaltspunkt: der Sitz des Corpus lut. Bei Fall 3 war der Gang noch erhalten, aber stenosirt, und das Ei stammte aus dem Eierstock des verkümmerten Horns. Hier wird die Befruchtung auf dem. gewöhnlichen Wege erfolgt sein.

Die Beschwerden, welche im Verlaufe einer Schwangerschaft im rudimentären Nebenhorn auftreten, sind im Anfange, wie es auch diese Fälle zeigen, unerheblich und geben uns nur das Bild der unsicheren Anzeichen einer Schwangerschaft. Schreitet die Schwangerschaft weiter, wie in Fall 1 und 3, dann sind auch die Störungen erheblicher - heftige Schmerzen im Unterleibe und häufiges Erbrechen -, doch sind auch diese Beschwerden nicht so in die Augen springend, dass man aus ihrem Bestehen bestimmte diagnostische Schlüsse ziehen könnte. Nur die Ausstossung hautähnlicher Stücke, wie bei Fall 1 (anscheinend auch bei Fall 2) liesse den Verdacht einer extrauterinen Gravidität aufkommen.

Bei Fall 1 und Fall 3 ist das seltene Vorkommniss hervorzuheben, dass beide den Endtermin der Schwangerschaft ohne Ruptur erreieht haben. Fall 1 gelangte sogar erst 8 Monate nảch Ende der Schwangersehaft zur Operation.

Gewöhnlich entsteht eine Ruptur im 3.-5. Monat, wie es uns Fall 2 und 4 zeigt. Beide Fälle haben insofern eine gewisse Aehnlichkeit, als bei beiden eine mechanische Ursache zu Grunde lag, welehe zur Operation geführt hat. Bei Fall 2 führte ein unglücklicher Sturz zur Blutung mit: nachfolgenden Schmerzen, bei Fall 4 starke körperliche Anstrengung zur Ruptur. Aehnliche Anlässe sind auch bèi anderen Fällen häufig beobachtet worden.

Bei allen 4 Fällen hörte mit dem Beginn der Schwangerschaft die Regel auf. Die Bildung einer Decidua in dem gesunden Horn ist nur bei Fall 1 (vielleicht auch Fall 2) beob- 
achtet worden. Thr Abgang erfolgte bei Fall 1 mit dem Ende der Schwangerschaft, bei Fall 2 nach dem unglücklichen Sturze.

Der'Sitz der Ruptur soll sich nach früheren Beobachtungen gewöhnlich an der höchsten Kuppe des Fruchtsacks befinden. Da in unseren Fällen jedoch nur 1 Fall (Fall 4) zur Ruptur gekommen ist und zwar so, dass der Fruchtsack dicht am Stiel circulär abgerissen ist, so können diese 4 Fälle zur Unterstützung obiger Erfahrung wenig Nutzen finden, doch möchte bervorgehoben werden, dass in den ersten 3 Fällen die Wandstärke des Fruchtsacks an der Spitze am geringsten war.

Sind die wahrscheinlichen oder sicheren Zeichen einer Schwangerschaft vorhanden, ist neben dem Uterus eine Geschwulst zu fühlen, so liegt der Hauptwerth der Diagnose auf dem Verbindungsstrang zwischen dem wirklichen Uterus und dem Fruchtsack, dem schwangeren rudimentären Horn. Die Dicke und Festigkeit dieses Stranges, der Ausgang desselben von der Gegend des inneren Muttermundes des Uterus findet man fast bei keiner anderen ektopischen Schwangerschaft. Von besonderem Werthe ist natürlich der Befund einer leeren Uterushöhle. Ein gewisses Gewicht ist auch auf den Abstand zwischen Uterus und Fruchtsack und auf eine beschränkte Beweglichkeit zu legen, wenn es sich um eine Schwangerschaft im rudimentären Horne handeln soll, da eine Schwangerschaft im Horn eines Uterus bicornis non atreticus oder septus bilocularis solche Gesichtspunkte nicht darbieten wird. Fühlt man neben oder auf dem Fruchtsack noch Gebilde, welche den Anhängen entsprechen, dann ist dieser Befund für die schon ziemlich sichere Diagnose eine Stütze mehr.

Ist die Diagnose gestellt, dann giebt es auch nur eine Therapie, und zwar die Operation, wie auch der Erfolg bei diesen 4 Fällen gelehrt hat. Wie schlecht sonst die Prognose zu stellen ist, das zeigt die Thatsache, dass Kehrer unter seinen Fällen $82 \mathrm{pCt}$. Mortalität hat. Eine Schwierigkeit liegt in der Abbindung des Stiels, dessen Blutstillung trotz grösster Mühe nicht gelingen kann und die den Operateur trotz seines conservativen Princips: nur den Fruchtsack abzutragen, in die Zwangslage versetzen kann, einen grösseren Eingriff zu machen und den Uterus zu exstirpiren, wie in Fall 1. Je kürzer und dicker der Stiel, um so grösser die Mühe.

Sämmtliche 4 Fälle konnten als geheilt entlassen werden. 
516 Krull, Vier Fälle von Schwangersch. im rudiment. Nebenhorn d. Uterus.

Wie sehr das Princip, möglichst conservativ bei der Operation vorzugehen und den Frauen die Fortpflanzungsmöglichkeit zu lassen, von Erfolg gekrönt war; das zeigen Fall 2 und 3, welche in hiesiger. Klinik später normale ausgetragene Kinder geboren haben.

Am Schlusse dieser Arbeit habe ich die angenehme Pflicht, meinem hochverehrten Chef, Herrn Geheimrath Leopold, für die Anregung zu der Arbeit und für die gütige Unterstützung meinen verbindlichsten Dank auszusprechen. 